«Charities » et association : une étude comparative France - Royaume-Uni du comportement des donateurs et des stratégies de collecte British charities and French nonprofits: A comparative study of donor behavior and fundraising in France and United Kingdom

\title{
Fanny Gérome
}

Numéro 318, octobre 2010

URI : https://id.erudit.org/iderudit/1020850ar

DOI : https://doi.org/10.7202/1020850ar

Aller au sommaire du numéro

\section{Éditeur(s)}

Association Recma

\section{ISSN}

1626-1682 (imprimé)

2261-2599 (numérique)

Découvrir la revue

\section{Citer cet article}

Fanny Gérome (2010). "Charities » et association : une étude comparative France - Royaume-Uni du comportement des donateurs et des stratégies de collecte. Revue internationale de l'économie sociale, (318), 39-57.

https://doi.org/10.7202/1020850ar

\section{Résumé de l'article}

La loi sur les charities est beaucoup plus ancienne que la loi de 1901. Ces organisations se limitent à des domaines plus étroits que les associations françaises. Elles n'ont également pas les mêmes arguments fiscaux pour inciter les donateurs à réaliser un don. Les Britanniques donnent beaucoup plus que les Français et sont plus nombreux à donner. La concentration des revenus de l'ensemble du secteur auprès de quelques organisations est plus importante au Royaume-Uni qu'en France. Il existe dans ces deux pays une forte concurrence à la recherche de financement, principalement due à l'augmentation de la taille des organisations, à l'intégration de nouveaux entrants dans le secteur et à la baisse ou à la stagnation des financements publics. Les outils de collecte sont nombreux et divers de façon à toucher un public de plus en plus large. L'innovation est constante en la matière. Pour satisfaire leurs donateurs, les associations et les charities doivent être concrètes dans leur communication auprès du public. 


\section{" CHARITIES » ET ASSOCIATIONS: UNE ÉTUDE COMPARATIVE FRANCE - ROYAUME-UNI DU COMPORTEMENT DES DONATEURS ET DES STRATÉGIES DE COLLECTE}

* Chargée de mission au Cnar Financement (www.solfia.org). Mél. : fannyg@franceactive.org.

\author{
par Fanny Gérome*
}

La loi sur les charities est beaucoup plus ancienne que la loi de 1901. Ces organisations se limitent à des domaines plus étroits que les associations françaises. Elles n'ont également pas les mêmes arguments fiscaux pour inciter les donateurs à réaliser un don. Les Britanniques donnent beaucoup plus que les Français et sont plus nombreux à donner. La concentration des revenus de l'ensemble du secteur auprès de quelques organisations est plus importante au Royaume-Uni qu'en France. Il existe dans ces deux pays une forte concurrence à la recherche de financement, principalement due à l'augmentation de la taille des organisations, à l'intégration de nouveaux entrants dans le secteur et à la baisse ou à la stagnation des financements publics. Les outils de collecte sont nombreux et divers de façon à toucher un public de plus en plus large. L'innovation est constante en la matière. Pour satisfaire leurs donateurs, les associations et les charities doivent être concrètes dans leur communication auprès du public.

$\mathrm{D}$

epuis plusieurs années, les associations sont invitées à diversifier leurs ressources, en d'autres termes à développer les financements privés, afin qu'elles puissent anticiper et apporter une réponse aux changements des modalités de financement avec la puissance publique. En effet, même si les pouvoirs publics ne se désengagent pas du financement des associations, ils ont modifié en profondeur les relations qu'ils entretenaient avec elles. Les raisons en sont multiples: réduire les coûts, avoir le sentiment de payer le "juste prix", reprendre la main sur les projets (en en assurant l'initiative et la maîtrise), ne plus assumer seul le financement de l'intérêt général en permettant la participation d'autres acteurs: fondations, entreprises, particuliers.

Pour les associations, ces bouleversement marquent notamment la fin des subventions de fonctionnement, la multiplication des appels à projets, le passage de la subvention à la commande publique, un accroissement 
du nombre d'acteurs avec l'ouverture à la concurrence privée, des exigences différentes suivant les partenaires, ainsi que le développement de financements privés dans une logique soit de prestations de services, soit de dons et de mécénat.

Sur ce dernier point, les associations doivent s'interroger sur leur stratégie de développement du don et du mécénat, en prenant en compte l'état actuel du marché de la générosité et leur potentiel de développement. Pour cela, elles doivent se poser les questions suivantes: en France, quel est le marché de la générosité? Qui sont les donateurs? A qui donnent-ils? Pour quelle cause? Comment sont-ils sollicités? Quels sont les outils utilisés ou sous-utilisés et à quels publics s'adressent-ils? Quel est mon public de donateurs? Qui les sollicite déjà et comment, avec quels outils? Quelles sont leurs exigences? En tant que responsable associatif, quelle est ma capacité de mobilisation de ressources humaines et financières? Quels sont les outils adaptés à l'objet et à la taille de mon association? Pour quel budget?

Ces questions sont plus récentes en France que dans d'autres pays comme le Royaume-Uni, où la collecte de fonds est plus ancrée et où la générosité des habitants est plus forte.

Après un bref rappel de la vie associative outre-Manche, cet article se propose de dresser un panorama et de comparer le marché de la générosité et les outils de collecte utilisés en France et au Royaume-Uni.

\section{Présentation des « charities " au Royaume-Uni}

(1) Cette partie est tirée de NCVO, 2007. Ici sont prises en compte les organisations du voluntary sectorayant un poids économique significatif et répondant à des critères tels que l'indépendance, une forme constitutionnelle, etc. (soit majoritairement les charities). Données: mai 2004. Source: NCVO, Guide Star UK.

(2) Le fonctionnement et I'organisation des charities sont régis par le Charities Act de 2006.
Le terme anglais charity peut être traduit par "charité ", " aumône ", mais aussi par "œuvre caritative", " organisation qui vient en aide". Par commodité, nous parlerons de charities. Elles existent au RoyaumeUni depuis plus de quatre cents ans ${ }^{(1)}$. C'est le Charitable Uses Act qui a reconnu officiellement, en 1601, les domaines pouvant être considérées comme charitables. Aujourd'hui, ce statut est attribué par la Charities Commission à un organisme lorsque celui-ci respecte l'ensemble des critères prévus ${ }^{(2)}$. Son objet doit notamment répondre à l'une des quatre catégories suivantes: lutte contre la pauvreté, promotion de l'éducation, promotion de la religion ou autre action au profit de la communauté (par exemple la lutte contre le racisme, la réinsertion des délinquants, etc.). Il n'y a pas de charity type. Certaines sont importantes, d'autres petites. Certaines se limitent à une zone géographique, parfois extrêmement restreinte comme un quartier ou un village; d'autres ont une envergure nationale, voire internationale. Il est cependant intéressant de noter qu'il y a eu au Royaume-Uni, de 1991 à 2005, une forte augmentation des charities, passant de 98000 organisations déclarées à plus de 168000 . Depuis 1995 (arrivée au pouvoir du nouveau parti travailliste représenté par Tony Blair), le nombre de charities a augmenté de $40 \%$. Le nombre de structures ayant un budget supérieur à 1 million de livres a doublé, 
essor dû en partie à la croissance des organisations existantes et à l'intégration de nouveaux entrants.

\section{Financement et budget des " charities "}

Plus de la moitié $(58,4 \%)$ des charities ont un budget annuel inférieur à 10000 de livres; 48000 structures ont un budget annuel compris entre 10000 et 100000 livres, 19000 structures, entre 100000 et 1 million de livres, et 3000 structures, entre 1 million et 10 millions de livres; 300 structures excèdent les 10 millions de livres, alors que 20 majorcharities, ont un budget qui dépasse les 100 millions de livres à l'année, ce qui représente $10 \%$ des revenus du secteur.

On peut constater que plus de $70 \%$ des revenus (dons, subventions...; voir les tableaux 1 et 2) ne concernent que 3500 organisations (représentant seulement $2 \%$ des charities), avec au sommet une vingtaine de supercharities, ayant une forte longévité. Ces dernières ont des activités proches des domaines de la santé, du handicap, de l'aide aux enfants et de la lutte contre la pauvreté, reflétant des problèmes sociaux du pays.

Les financements publics restent la source de revenus prépondérante des charities, représentant 38,6 \% des financements contre 50,7 \% en France. Les fonds collectés auprès des particuliers sont également très importants et sensiblement équivalents aux fonds publics.

En France, toutes structures associatives confondues, les financements (CNRS-Matisse, 2007) proviennent à 49,3\% du privé (cotisations, $12,1 \%$; dons et mécénat, 4,9\%; recettes d'activité, 32,3\%) et à 50,7\% du public (subventions publiques, $34,3 \%$; ventes de prestations ou prix de journée, $16,4 \%)$.

\section{Tableau 1 \\ Origine des financements des associations en France et au Royaume-Uni (RU)}

\begin{tabular}{|c|c|c|c|c|c|c|}
\hline $\begin{array}{l}\text { Sources } \\
\text { de financement }\end{array}$ & $\begin{array}{l}\text { Revenus } \\
\text { marchands }\end{array}$ & & $\begin{array}{l}\text { Revenus } \\
\text { non march }\end{array}$ & & Total & \\
\hline Pays & France & RU & France & RU & France & RU \\
\hline Public & $16,4 \%$ & $23,9 \%$ & $34,3 \%$ & $14,7 \%$ & $50,7 \%$ & $38,6 \%$ \\
\hline Privé & $32,3 \%$ & $32 \%$ & $17 \%$ & $29,4 \%$ & $49,3 \%$ & $61,4 \%$ \\
\hline Total & $48,7 \%$ & $55,9 \%$ & $51,3 \%$ & $44,1 \%$ & $100 \%$ & $100 \%$ \\
\hline
\end{tabular}

* Pour le détail des revenus marchands et non marchands, se reporter au tableau 2, en page suivante. 
Tableau 2

Source et répartition des revenus des organisations au Royaume-Uni, 2004-2005

\begin{tabular}{|c|c|c|c|}
\hline \multirow{2}{*}{ Source des revenus } & \multicolumn{3}{|l|}{ Type de revenus } \\
\hline & Revenus marchands & Revenus non marchands & Total \\
\hline Financements publics & $\begin{array}{l}23,9 \% \\
\text { Vente de prestations... }\end{array}$ & $\begin{array}{l}14,7 \% \\
\text { Subventions, loterie... }\end{array}$ & $38,5 \%$ \\
\hline Particuliers & $\begin{array}{l}12 \% \\
\text { Paiement des biens } \\
\text { et services fournis... }\end{array}$ & $\begin{array}{l}23,5 \% \\
\text { Dons, legs, Gift Aid } \\
\text { et cotisation sans contrepartie }\end{array}$ & $35,5 \%$ \\
\hline $\begin{array}{l}\text { Associations } \\
\text { et fondations }\end{array}$ & $\begin{array}{l}6 \% \\
\text { Sous-traitance }\end{array}$ & $\begin{array}{l}4,6 \% \\
\text { Subventions des fondations... }\end{array}$ & $10,6 \%$ \\
\hline Entreprises & $\begin{array}{l}2,5 \% \\
\text { Parrainage... }\end{array}$ & $\begin{array}{l}1,3 \% \\
\text { Mécénat }\end{array}$ & $3,9 \%$ \\
\hline Autres & $\begin{array}{l}-3,7 \% \\
\text { Revenus des filiales } \\
\text { commerciales (charity shops) } \\
\bullet \text { 7,8\% } \\
\text { Locations, intérêts financiers... }\end{array}$ & & $11,5 \%$ \\
\hline Total & $55,9 \%$ & $44,1 \%$ & $100 \%$ \\
\hline
\end{tabular}

Sur les sources de financement (public-privé), deux tendances proches peuvent être observées dans les pays étudiés.

Au Royaume-Uni, les financements publics sont attribués davantage pour le paiement de prestations: c'est ce que certains appellent la «charitisation " des services publics. Les charities bénéficient par le fait de moins de subventions. En France, les modalités de conventionnement avec les pouvoirs publics évoluent également dans ce sens. Les subventions pour des projets précis, le passage à la commande publique marquent une volonté de reprise de l'initiative par les collectivités et s'insèrent dans une logique de prestation de services (Juris Edition, 2010).

La part des dons des particuliers est pour les charities nettement supérieure à celle des structures françaises. Au regard des financements des associations en France sur les six dernières années, on peut cependant observer une nette augmentation des financements privés. La part concernant le don et le mécénat dans les ressources des associations représente $5 \%$ des budgets totaux (Juris Edition, 2010). 
Tableau 3

Source des revenus suivant la taille de l'organisation, 2004-2005

\begin{tabular}{|l|r|r|r|r|r|r|}
\hline Budget & $\begin{array}{r}\text { Inférieur } \\
\text { à } 10000 £\end{array}$ & $\begin{array}{r}\text { Entre } 10000 \\
\text { et } 100000 £\end{array}$ & $\begin{array}{r}\text { Entre } 100000 \\
\text { et } 1 \text { million } £\end{array}$ & $\begin{array}{r}\text { Entre } 1 \text { million } \\
\text { et } 10 \text { millions } £\end{array}$ & $\begin{array}{r}\text { Supérieur } \\
\text { à } 10 \text { millions } £\end{array}$ & Total \\
\hline Financement public & $17 \%$ & $35 \%$ & $49 \%$ & $40 \%$ & $33 \%$ & $39 \%$ \\
\hline Particuliers & $34 \%$ & $22 \%$ & $20 \%$ & $33 \%$ & $46 \%$ & $36 \%$ \\
\hline Associations & $23 \%$ & $25 \%$ & $18 \%$ & $10 \%$ & $5 \%$ & $11 \%$ \\
\hline Entreprises & $6 \%$ & $5 \%$ & $3 \%$ & $5 \%$ & $3 \%$ & $4 \%$ \\
\hline Autres & $21 \%$ & $13 \%$ & $10 \%$ & $11 \%$ & $12 \%$ & $11 \%$ \\
\hline Total & $100 \%$ & $100 \%$ & $100 \%$ & $100 \%$ & $100 \%$ & $100 \%$ \\
\hline
\end{tabular}

Les fonds collectés auprès des particuliers sont prépondérants dans deux cas seulement: pour les structures dont le budget est inférieur à 10000 livres et pour celles dont le budget est supérieur à 10 millions de livres. Une majorité de structures associatives du Royaume-Uni sont en effet de petites organisations avec des budgets faibles, qui fonctionnent en partie grâce au bénévolat et à la participation financière d'adhérents ou de bénévoles.

"Big is beautiful»: il est plus facile de collecter des fonds auprès des particuliers pour les grandes organisations connues et reconnues par le public (tableau 3). Il en de même pour les financements publics, qui se concentrent dans ces mêmes organisations dont les projets accompagnent l'action publique.

\section{Comment dépensent-elles?}

La concurrence face aux sources de financement s'étant accrue, les organisations attachent plus de poids à la transparence et à l'affectation du montant des dépenses. Près de $84 \%$ des sommes sont investies directement dans la réalisation des missions correspondant à l'objet social, et $16 \%$ dans la recherche de fonds (fundraising), la vente, le management et l'administration. Toutes organisations confondues, les frais de publicité et de recherche de fonds se sont élevés à près de 1,6 milliard de livres, dont 1,1 pour les organisations ayant un budget supérieur à 10 millions de livres. Pour ces dernières, ce type de charge représente $10,70 \% \mathrm{du}$ total des dépenses.

Assez logiquement, les 350 charities ayant les budgets les plus importants mettent donc en œuvre les techniques les plus sophistiquées pour faire appel à la générosité du public et réalisent ainsi les plus grosses collectes de dons auprès des particuliers. Lorganisation de collectes est plus difficile 
à réaliser pour les charities ne bénéficiant pas des mêmes moyens financiers et humains.

\section{Comparaison en matière d'incitation fiscale pour les dons}

Les charities et les associations n'ont pas les mêmes arguments fiscaux pour inciter les donateurs à réaliser un don. Le tableau 4 présente la synthèse des avantages fiscaux en France et au Royaume-Uni.

\section{Le "Gift Aid Scheme "}

Le Gift Aid Scheme permet aux contribuables de faire bénéficier les charities du remboursement de l'impôt correspondant à leurs dons. Seuls $34 \%$ des donateurs utilisent le Gift Aid pour au moins l'un de leurs dons. Il s'agit d'un manque à gagner important pour les charities, qui pourraient bénéficier d'un remboursement de la part du gouvernement sur

\section{Tableau 4 Les incitations fiscales à la générosité}

\begin{tabular}{|c|c|c|}
\hline & France & Royaume-Uni* \\
\hline $\begin{array}{l}\text { Les dons } \\
\text { des particuliers }\end{array}$ & $\begin{array}{l}\text { Lorsque le donateur réalise un don, il bénéfi- } \\
\text { cie d'une réduction d'impôt de } 66 \% \text { (75 \% } \\
\text { dans certains cas), dans la limite de } 20 \% \\
\text { du revenu imposable. Si le don dépasse ce } \\
\text { plafond, l'excédent peut être reporté et étalé } \\
\text { sur cinq ans. Il s'agit ici d'une volonté poli- } \\
\text { tique de favoriser les financements privés en } \\
\text { incitant les donateurs à réaliser un don ou à } \\
\text { augmenter le montant donné. Les associa- } \\
\text { tions ne bénéficient pas de remboursement } \\
\text { financier du Trésor public }\end{array}$ & $\begin{array}{l}\text { Lorsque les donateurs sont imposables } \\
\text { sur leurs revenus, le mécanisme du Gift } \\
\text { Aid Scheme permet aux charities de récu- } \\
\text { pérer la part d'impôt payée par le dona- } \\
\text { teur (avec son accord). Sur un don de } \\
100 \text { livres fait par un donateur imposé } \\
\text { à } 22 \% \text { sur ses revenus, par exemple, } \\
\text { une charity pourra récupérer auprès de } \\
\text { l'administration fiscale un complément } \\
\text { de } 28,20 \text { livres (part d'impôt déjà payée } \\
\text { par le donateur). Le don effectif sera } \\
\text { donc égal à } 128,20 \text { livres }\end{array}$ \\
\hline $\begin{array}{l}\text { Les legs } \\
\text { et les donations }\end{array}$ & $\begin{array}{l}\text { L'association, sous certaines conditions, peut } \\
\text { bénéficier d'une exonération totale des droits } \\
\text { de succession et de donation. Dans le cas } \\
\text { d'une donation à certains organismes (asso- } \\
\text { ciations reconnues d'utilité publique, struc- } \\
\text { tures de l'insertion par l'activité économique, } \\
\text { entreprises adaptées, etc.), le donateur peut } \\
\text { réduire le montant taxable pour le calcul de } \\
\text { l'ISF, à hauteur de } 75 \% \text { du montant de la } \\
\text { donation et dans la limite de } 50000 \text { euros }\end{array}$ & $\begin{array}{l}\text { Les legs et les donations à une charity } \\
\text { sont exemptés de droits de succession }\end{array}$ \\
\hline $\begin{array}{l}\text { Les dons } \\
\text { des entreprises }\end{array}$ & \multicolumn{2}{|c|}{$\begin{array}{l}\text { Les charities comme les associations peuvent bénéficier de dons de la part d'entreprises. } \\
\text { Ces dernières bénéficient également d'une réduction d'impôt (dans certaines limites) }\end{array}$} \\
\hline
\end{tabular}


(3) Les données de cette souspartie sont tirées, pour la France, de: Cerphi, 2005, 2006, 2009; Fondation de France, 2008; et, pour le Royaume-Uni, de: NCVO. CAF, 2004-2005; NCVO, 2007; NCVO-CAF, 2004-2005.

(4) Selon les statistiques de la Charity Commission de 1997 à 2003. le montant de l'impôt déjà payé par le donateur. Pour actionner le Gift Aid, le donateur doit juste donner son accord. Il s'agit souvent d'une simple case à cocher sur le formulaire de don. Est-ce un problème de communication de la part des charities vis-à-vis de leurs donateurs?

La question peut également être soulevée en France. En effet, de nombreux Français n'utilisent pas l'incitation fiscale. Selon une enquête menée par la Sofres pour l'Union nationale des organismes faisant appel à la générosité du public (Unogep) en mai 2004 (données reprises dans Cerphi, 2005), $90 \%$ des Français déclaraient savoir qu'il existait un système de réduction d'impôt pour les dons, mais seul un sur dix le connaissait avec précision. L'une des hypothèses émises par le Cerphi $(2005,2006)$ est que les associations n'ont peut-être pas communiqué assez clairement sur le dispositif. A ce titre, l'étude déconseille de "mélanger générosité et fiscalité " et propose d'orienter la communication non pas sur l'économie pouvant être réalisée, mais sur l'efficacité du don: "Mon pouvoir de donner plus la bonification de l'Etat égale mon pouvoir d'agir. "

\section{Le comportement des donateurs britanniques et français}

Au Royaume-Uni, le déclin de l'Etat-providence, marqué par d'importantes vagues de privatisation dans les années 80 avec Margaret Thatcher (Premier ministre de 1979 à 1990), a conduit au développement et à l'augmentation du nombre de charities ${ }^{(3)}$. Ainsi, 7000 nouvelles charities sont enregistrées chaque année auprès de la Charity Commission depuis le milieu des années $90^{(4)}$.

Avec l'augmentation du nombre de structures, la concurrence à la collecte de fonds s'est particulièrement accrue. Ce secteur est de plus en plus dominé par les majors, qui utilisent les techniques de marketing pour la collecte de fonds et sont capables d'attirer davantage de fonds que les autres organisations (Sargeant, 2005). Aujourd'hui, les revenus sont concentrés sur un petit nombre de structures: $2 \%$ se partagent $70 \%$ des revenus au Royaume-Uni, contre $2 \%$ des structures pour $55 \%$ des revenus en France (CNRS Matisse, 2007).

A l'instar de ce que l'on observe au Royaume-Uni, le secteur associatif français semble confronté à des difficultés croissantes pour obtenir des financements publics et nombreuses sont les organisations s'interrogeant sur le financement des parties non prises en charge par les pouvoirs publics. Pour cela, elles développent depuis quelques années des activités de collecte de fonds auprès des particuliers et des entreprises. Il peut être intéressant de comparer le marché de la générosité et les outils de collecte utilisés en France et au Royaume-Uni, afin de comprendre leur évolution et d'en tirer des enseignements.

\section{Qui donne quoi?}

\section{En France}

Les dons déclarés à Bercy s'élèvent en 2005 à 1,555 milliard d'euros, auxquels il faut ajouter les dons non déclarés. Si le montant des dons connait 
une légère hausse régulière, le nombre de donateurs reste stable (18 millions de personnes environ; voir le tableau 5 , en page suivante). Le don moyen annuel est de 125 euros, répartis en moyenne sur trois dons dans l'année (Ifop pour l'observatoire de la Fondation de France, mai 2006). En 2006, la typologie des donateurs français se présentait de la façon suivante (Cerphi, 2006):

- 20 à $23 \%$ de donateurs réguliers par chèque, virement ou prélèvement automatique;

- 20 à $22 \%$ de donateurs réguliers en espèces, de la main à la main ou par SMS;

- $21 \%$ de donateurs occasionnels, réagissant aux sollicitations ponctuelles ;

- $36 \%$ de personnes ne donnant pas du tout (dont $11 \%$ ayant donné pour la première fois lors du tsunami qui a frappé l'Asie du Sud-Est en décembre 2004).

Différents facteurs sont repérables dans le comportement des donateurs français (Fondation de France, 2003):

- l'âge (contrairement aux Britanniques, chez les Français le nombre et l'importance des dons augmentent avec l'âge);

- la pratique religieuse;

- le genre (les femmes donnent légèrement plus que les hommes);

- la richesse de la région d'habitation;

- les caractéristiques socioprofessionnelles (68\% des cadres supérieurs déclarent faire un don, contre $41 \%$ d'ouvriers). Il faut souligner que, proportionnellement à leur revenu, les foyers les plus modestes donnent plus que les foyers riches.

Selon le baromètre 2008 de la générosité en France (Fondation de France, 2009), les Français sont attirés par les dons par chèque et par virement. Ils sont toutefois nombreux à faire des dons de nourriture (près de $13 \%)$, de vêtements (24\%) et d'autres dons en nature (27\%; don de temps, par exemple).

\section{Au Royaume-Uni}

Vingt-huit millions de Britanniques sont donateurs. Le montant total des dons pour 2005-2006 est estimé à 8,9 milliards de livres, chiffre comparable à celui de l'année précédente (8,2 milliards de livres). En 2005-2006, le don moyen annuel est de 324 livres, en augmentation de presque $10 \%$ sur un an.

L'Institute for philanthropy dressait en 2004 cette typologie des donateurs britanniques:

- $12 \%$ d'investors ("investisseurs »), qui donnent plus de 120 livres (parmi eux, $3 \%$ donnent plus de 500 livres);

- $58 \%$ de contributors (" contributeurs »), qui donnent entre 5 et 120 livres;

- $30 \%$ de bystanders (" spectateurs »), qui donnent moins de 5 livres par an.

Là aussi, des constantes sont identifiables dans le profil des donateurs:

- l'âge (entre 25 et 65 ans);

- les études et la catégorie socioprofessionnelle supérieures; 
- les croyances et les pratiques religieuses;

- le genre (davantage de femmes donnent, mais le volume des dons masculins reste supérieur);

- la région d'habitation. Ainsi, le Sud-Ouest et l'Ecosse ont le plus fort pourcentage de donateurs, représentant $60 \%$ de la population totale, alors que les Londoniens, région la plus riche du royaume, ne sont que $51 \%$ à donner, avec certes le plus fort montant de don mensuel (22 euros). Le moyen utilisé pour donner va dépendre du mode de collecte choisi par l'organisation. Le moyen le plus populaire reste le don en espèces, de la main à la main, mais ce sont les prélèvements automatiques et les chèques qui collectent les montants les plus élevés.

Les Britanniques sont, au contraire des Français, très peu nombreux à réaliser des dons de nourriture (4\%). Selon Mme Vian, secrétaire générale de la Fédération européenne des banques alimentaires, ce comportement s'explique par le fait quau Royaume-Uni "donner de la nourriture est considéré comme une assistance peu favorable à la personne bénéficiaire. L'aide alimentaire directe n'est donc pas communément envisagée, ni par les associations dans leurs actions, ni par la population dans sa générosité" (Institute for philanthropy, 2004). En revanche, les Britanniques sont nombreux à faire des dons de vêtements $(30 \%)$ et d'autres dons en nature (34\%). On peut penser qu'ils sont beaucoup plus sollicités par les charities, notamment par le biais des charity shops (lire infra).

\section{Tableau 5}

\section{Tendances et évolutions}

\begin{tabular}{|c|c|}
\hline France & Royaume-Uni \\
\hline $\begin{array}{l}\text { - Le nombre de donateurs reste stable } \\
\text { - L'effort en matière de dons en argent est } \\
\text { porté très fortement par les donateurs de plus } \\
\text { de } 50 \text { ans } \\
\text { - Si les ménages aisés réalisent des dons plus } \\
\text { importants en valeur, ce sont les ménages } \\
\text { modestes qui consacrent la part de leur revenu } \\
\text { la plus importante aux dons } \\
\text { - Les organisations associatives communi- } \\
\text { quent davantage d'informations financières } \\
\text { (influence du Comité de la Charte**) }\end{array}$ & $\begin{array}{l}\text { - Croissance spectaculaire du nombre de } \\
\text { grandes fortunes au Royaume-Uni } \\
\text { - Apparition d'un nouveau profil de donateur, } \\
\text { plus jeune, prêt à investir davantage } \\
\text { - De plus en plus de personnes veulent donner } \\
\text { de leur vivant } \\
\text { - Les donateurs demandent à connaîtrel'impact } \\
\text { de leurs dons (nombre de personnes aidées...) } \\
\text { - Les donateurs sont vigilants sur la trans- } \\
\text { parence financière (utilisation de l'argent de } \\
\text { façon efficiente et efficace) }\end{array}$ \\
\hline
\end{tabular}

* Source: www.philanthropyuk.org.

** Le Comité de la Charte, créé il y a vingt ans, est un organisme d'agrément et de contrôle des associations et des fondations faisant appel à la générosité du public qui s'engagent volontairement à respecter une charte de déontologie et à se soumettre à des contrôles. Plus d'informations sur www.comitecharte.org. 
(5) Ce système permet aux salariés de retenir directement sur leur salaire les dons qu'ils souhaitent effectuer. Les règles en matière de fiscalité sont identiques au Gift Aid Scheme (pour les dons ponctuels). Les impôts sur le revenu étant prélevés à la source au Royaume-Uni, cette procédure simplifie le travail de la charity, qui n'a pas besoin de réclamer au Trésor public le remboursement de l'impôt déjà payé. (6) Selon le Cerphi, les legs représenteraient 600 millions d'euros pour le secteur caritatif et la recherche médicale.

\section{Comment donne-t-on?}

Il y a plusieurs manières de donner: par un simple don (avec utilisation ou non du Gift Aid Scheme pour le Royaume-Uni et avec l'utilisation ou non de la réduction d'impôt en France); par un don en nature ou de vêtements; par des dons réguliers (par prélèvement ou en utilisant le Payroll Giving Scheme ${ }^{(5)}$ ) ; en devenant membre de l'organisation en payant une cotisation annuelle; par un legs. La pyramide des dons (Norton, 2007) peut être représentée par le schéma 1.

En France, les legs aux associations représenteraient 1 milliard d'euros. Ce montant, à prendre avec précaution, car il est difficile de répertorier de façon exhaustive le montant des legs ${ }^{(6)}$, est en constante augmentation et a été multiplié par dix sur ces dix dernières années. Les testateurs sont généralement des femmes, autour de 79 ans, veuves et sans héritiers. Au Royaume-Uni, les legs représentent 1,6 milliard de livres, soit $6 \%$ des revenus totaux des charities.

\section{Quelles causes soutient-on?}

Au Royaume-Uni, les causes les plus populaires (relativement au nombre de donateurs) sont la recherche médicale (lutte contre le cancer), les enfants et les jeunes, les hôpitaux, l'aide internationale, les animaux, les personnes handicapées et les organisations religieuses. La recherche médicale représente à elle seule $19 \%$ du total des dons; viennent ensuite les organisations religieuses avec $16 \%$, l'aide internationale avec $13 \%$ et les hôpitaux avec $12 \%$. Au cours des vingt-cinq dernières années, le domaine culturel a gagné en popularité, en grande partie grâce au financement de la Loterie nationale.

Pour la France (Tchernonog, 2007; Fondation de France, 2009), les dons et le mécénat représentent $26 \%$ des budgets des associations d'action

\section{Schéma 1 La pyramide des dons}

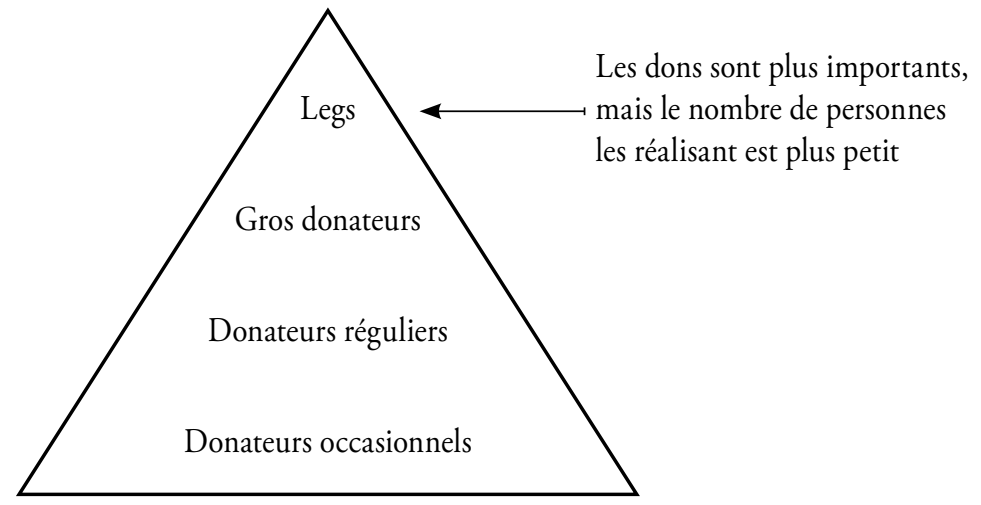


(7) Trente individus auraient donné l'équivalent de $15 \%$ des 8,9 milliards de livres de dons collectés en 2006 (Fortson D., "Hedge funds get the bug for benevolence ", The Independent, 20 mai 2007, www.fundraising co.uk).

(8) " How much do people give to charity ", site Internet de la BBC 8 décembre 2004. humanitaire, $7 \%$ de celles œuvrant pour la défense des droits et des causes et environ $6 \%$ des associations sportives et culturelles. Si dans ces secteurs les outils et les appels de collecte se sont développés, les domaines d'intervention soutenus par le plus grand nombre de donateurs (tous types de don confondus) sont l'aide aux personnes en difficulté, la santé, la recherche, notamment médicale, et l'aide humanitaire internationale. Avec la crise, on constate un recentrage des dons sur des actions plus proches des préoccupations directes des Français: entre 2007 et 2008 s'accroît le nombre de donateurs pour l'aide aux personnes en difficulté, alors que l'aide au tiers monde connaît une légère baisse.

Il y a de fortes disparités entre la générosité britannique et la générosité française, à commencer par le montant total des dons tout simplement (excepté pour le tsunami de décembre 2004) ou encore l'âge des donateurs (retraites plus faibles, salaires des cadres plus élevés au Royaume-Uni...). Ces différences s'expliquent par un ensemble de critères historiques, religieux, mais aussi sociologiques. Le Royaume-Uni a une longue tradition philanthropique et l'on constate que la culture du don et des charities est fortement partagée par la population. Certains pensent que le RoyaumeUni pourrait faire mieux et citent avec nostalgie les grands philanthropes. Est-ce un retour des choses? Le Royaume-Uni concentre aujourd'hui une forte population de milliardaires et voit fleurir de nouveaux grands projets philanthropiques ${ }^{(7)}$...

Enfin, si le marché de la générosité se développe en France depuis plus de dix ans, 2003 a marqué au Royaume-Uni la première baisse de la collecte de fonds depuis $1996^{(8)}$.

\section{Les différents outils de collecte de fonds auprès des particuliers}

(9) Cette partie est tirée des ouvrages suivants: GollopelMorvan et alii, 2008; Le Péchon, Rieunier 2008; Bruce, 2005).
Après avoir évoqué les spécificités de la France et de la Grande-Bretagne concernant la structure de leurs associations et le comportement de leurs donateurs respectifs, nous présentons les différents outils de collecte auprès des particuliers ${ }^{(9)}$. Au-delà du détail des modalités de collecte, se dessine une tendance à l'uniformisation des pratiques consistant pour les organisations, d'une part, à rendre très concret l'acte de don et, d'autre part, à valoriser leurs donateurs. On distingue six grandes manières de collecter des dons auprès de particuliers: le marketing direct, celui des donations et des legs, les collectes au travail, les collectes locales, les ventes charitables et l'organisation d'événements.

\section{Le marketing direct}

Le marketing direct se caractérise par une approche personnalisée et à distance. Pour la collecte de fonds auprès de secteur associatif, il peut revêtir plusieurs formes.

Papier (mailing)

Il s'agit de courriers électroniques, de lettres, de catalogues ou de colis 
envoyés de façon personnalisée. Ils permettent d'informer, de sensibiliser les nouveaux donateurs aux actions réalisées par l'organisme. Ce moyen de collecte est particulièrement coûteux, avec un retour de $3 \%$ par envoi. En France, le mailing reste le moyen de collecte privilégié: $80 \%$ des dons viennent de cette sollicitation. Les plus de 50 ans, représentant la part la plus importante des donateurs en France, sont particulièrement sensibles à ce type de collecte. On constate cependant une sur-sollicitation du public avec cet outil (vente des fichiers de donateurs entre associations, donateurs saturés, affaiblissement du rendement).

\section{Téléphone (" phoning ")}

Il est utilisé pour des actions de prospection et de fidélisation. Il peut être coûteux pour une association qui ferait appel à une société spécialisée pour faciliter le ciblage du public et gérer les appels.

\section{Publicité (télévision, radio, affichage métro...)}

Les publicités sont parfois associées à des célébrités. Le coût varie selon le média, et certaines associations bénéficient d'espaces publicitaires gratuits.

\section{Nouvelles technologies}

Il est souvent proposé de donner par téléphone, par SMS, pour faciliter les dons spontanés. Internet et les SMS sont plutôt des outils liés à l'urgence. Ils permettent aux internautes de donner rapidement. Par ailleurs, le site Internet peut être un moyen de fournir de l'information sur l'organisation. Oxfam propose par exemple aux internautes différents moyens d'aider l'organisation: don d'argent, de temps...

\section{Le marketing du legs et des donations}

Il peut s'agir d'un bien tel qu'un tableau, d'une somme d'argent ou d'un immeuble. La démarche peut s'organiser auprès des notaires, ou legacy solicitors, des donateurs, réguliers ou non, ou encore du public. Dans les deux pays, le marketing du legs est le parent pauvre de la collecte de fonds. Il s'agit sans doute d'un des outils offrant le plus de possibilités de développement. En France comme en Angleterre, le marché du legs est très concentré sur peu d'organisations. Pour les organisations traitant de problématiques liées à l'enfance maltraitée, aux personnes handicapées (malvoyantes, paralysées) ou au sauvetage des personnes en mer ou encore pour les charities contre le cancer, les legs représentent parfois $40 \%$ ou plus de leur revenu. RNIB, une charity caritative, écrit deux fois par an depuis plus de cinquante ans à tous les notaires du pays. Les legs représentent $70 \% \mathrm{de}$ l'ensemble de ses revenus non marchands.

\section{La collecte au travail}

Il existe de nombreuses méthodes de collecte au travail: journée où les salariés s'habillent avec une couleur précise (Green Day pour l'environnement, par exemple), challenge entreprise comme une course associant les salariés 
et parfois les clients, etc. Il s'agit d'un bon moyen pour toucher les moins de 65 ans. Ces méthodes de collecte au travail (ou dans les écoles) sont très largement développées au Royaume-Uni. Les courses en entreprise ou à l'école commencent à apparaître en France.

\section{La collecte locale}

Elle peut se présenter sous trois formes: le face-à-face, la collecte dans la rue avec un tronc et la participation à des événements locaux.

\section{Face-à-face}

La méthode la plus visible est le street marketing (" collecte de rue »). Il s'agit de groupes de jeunes qui, dans la rue, sensibilisent les passants à une cause et leur demandent de soutenir financièrement une organisation (par prélèvement automatique). Les objectifs sont de faire connaître les actions de cette organisation et de collecter des fonds. Cet outil semble être adapté pour toucher des donateurs plus jeunes et pour être vu par le public. Il présente toutefois des limites: il est coûteux, risque de porter préjudice à l'image de l'association auprès des personnes sur-sollicitées et ne permet pas de fidéliser les donateurs. Au Royaume-Uni, toutes les grandes organisations humanitaires et de santé, comme Research Cancer, utilisent cet outil, mais elles ont commencé à enregistrer une baisse de performance. En France, la majorité des organisations utilisant le street marketing sont des associations humanitaires. Cet outil leur permet d'avoir accès à des profils de donateurs jeunes, public particulièrement sensible aux actions internationales.

Avec le porte-à-porte (ou door-to-door), qui consiste à aller frapper à la porte des gens avec un prospectus et un formulaire de don, l'objectif est double: trouver à la fois de nouveaux donateurs et de nouveaux bénévoles. Ce peut être un bon outil pour les associations ayant une action au niveau local. Au Royaume-Uni, la collecte door-to-door pourrait pallier la baisse de rendement du street marketing.

\section{Collecte dans la rue avec un tronc}

Le static collecting devices consiste à organiser une collecte à un endroit fixe, généralement à proximité d'un endroit public (magasin, théâtre). Pour retenir l'attention, des sifflets ou des cloches sont souvent utilisés. L'Armée du Salut offre sans doute le meilleur exemple de ce type de

(10) L'histoire raconte qu'en 1914 des terres situées en Picardie et en Flandres furent tellement remuées par les soldats pendant les batailles que des coquelicots fleurirent comme jamais. La Royal British Legion décida donc d'utiliser le coquelicot comme emblème et créa une usine où des blessés de guerre fabriquaient dès 1922 des coquelicots en papier, vendus au profit des anciens combattants autour du 11 novembre. collecte, car ses collecteurs utilisent une clochette reconnaissable dans n'importe quel pays.

Le street collections and flag day constitue une autre forme de collecte de rue. Un drapeau ou un autre petit cadeau est donné aux personnes qui réalisent un don au cours d'une journée nationale annuelle. L'une des journées nationales les plus connues au Royaume-Uni est sans doute le Poppy Day, ou "journée du Coquelicot» ${ }^{(10)}$. En 2006, les journées de collecte ont rapporté près de 25 millions de livres. La Royal British Legion bénéficie d'un très important réseau sur tout le territoire et de nombreux 
partenariats. A titre indicatif, la journée de collecte de la Croix-Rouge génère en France 4 millions d'euros.

\section{Evénements locaux}

Course, kermesse et loterie peuvent être un excellent moyen de collecte de fonds, notamment pour une organisation ayant une action locale. En France, les associations peuvent organiser six manifestations de ce genre, ouvertes au public et exonérées d'impôt.

\section{Les ventes charitables}

"Charity shops"

Les charity shops, ou "boutiques charitables", sont des points de vente d'articles d'occasion donnés pour recueillir des fonds. Il existerait plus de 7000 boutiques charitables au Royaume-Uni, dont plusieurs centaines à Londres. Elles représentent près de 300 organisations et 12000 bénévoles s'en occupent, certains magasins étant même gérés exclusivement par des bénévoles (www.charityshops.org.uk). Toutefois, ces boutiques souffrent de la concurrence des ventes aux enchères sur Internet. Cette concurrence semble avoir eu pour conséquence directe une baisse de la qualité des dons. Certaines charities ont donc développé de nouveaux magasins spécialisés dans la vente de livres, de disques, de meubles d'occasion... D'autres organisations ont créé des boutiques en ligne sur leur site Internet. Oxfam compte plus de 800 boutiques.

\section{Ventes aux enchères}

Elles sont très populaires au Royaume-Uni et le deviennent depuis peu en France. Le célèbre site Internet de vente aux enchères eBay propose sur sa version anglaise une page consacrée aux ventes au profit exclusif de charities.

\section{Sites dédiés aux dons pour les "charities "}

Sur ces sites, les internautes disposent d'une liste d'organisations et n'ont plus qu'à choisir en fonction de la cause défendue. Les sites les plus connus au Royaume-Uni sont www.justgiving.com et www.goodgifts. org. Ce dernier propose de faire un don comme cadeau d'anniversaire, de mariage... Il invite à des achats très concrets, comme un cours de danse pour encourager les personnes âgées à rester en forme.

\section{Les événements...}

\section{... glamour}

Il s'agit ici du moyen de collecte le plus glamour: bals, dîners... Outre la collecte de fonds, ces événements permettent de donner une image dynamique de l'organisation, favorisent la participation des bénévoles et sensibilisent le public à une cause. En France, ces manifestations sont encadrées, mais au Royaume-Uni toutes les causes peuvent être défendues par ce biais (établissements scolaires, établissements de soins, recherche...). 
(11) Le Royaume-Uni félicite les meilleurs collecteurs du pays au travers d'une cérémonie avec remise de prix (voir www. nationalawards.org.uk).
C'est ainsi que le lycée français de Londres réalise chaque année une soirée de gala au profit de deux charities qui soutiennent l'établissement.

\section{... sportifs}

Les très grosses charities organisent toutes un événement sportif annuel de collecte de fonds (certaines associations françaises également). Marie Curie Cancer organise ainsi une course à vélo réunissant cinq cents personnes et se terminant à Varsovie (ville originaire de Marie Curie). La participation est d'environ 235 livres, auxquels il faut ajouter 1450 livres (plus de 2000 euros) de collecte auprès de sponsors.

\section{Uniformisation et valorisation}

Pour collecter des fonds, il existe une multitude d'outils plus ou moins efficaces selon l'âge des donateurs. Les moins de 50 ans préféreront le contact direct avec les bénévoles, par exemple, alors que les plus de 50 ans seront plus sensibles au mailing (Rieunier, 2006). Néanmoins, les charities comme les associations voient leurs pratiques s'homogénéiser en fonction du public visé. Certains publics sont d'ailleurs saturés par les échanges de fichiers des donateurs entre chercheurs de fonds et par la multiplication des sollicitations. Charities et associations sont donc à l'affût de nouvelles techniques ${ }^{(11)}$.

Devant cette saturation du marché, il est primordial de satisfaire les donateurs. Sept Français sur dix font globalement confiance aux actions réalisées par les associations, mais près de $85 \%$ des personnes interrogées (CSA, 2007) pensent que les associations doivent améliorer la qualité de l'information financière transmise à leurs financeurs et adhérents, mieux gérer leurs ressources et veiller au bon fonctionnement de leur gouvernance (AG et $\mathrm{CA}$ ). Pour que les donateurs puissent comprendre l'impact de leur don, les charities comme les associations sont donc de plus en plus concrètes dans leur collecte de fonds. Les exemples sont nombreux: "Pour 1 livre, on peut acheter un sachet de riz "; moins trivial, le musée du Tate Britain à Londres, par le biais de la charity The Art Fund, a proposé de faire des dons pour sauver une peinture célèbre de Rubens.

En général, des deux côtés de la Manche, les organisations informent régulièrement leurs donateurs. Certaines envoient le rapport annuel, leur propose de participer aux événements annuels (outils de collecte parfois), de signer des pétitions, de donner du temps pour l'organisation de manifestations... Le bénévolat est effectivement une ressource primordiale, même en ligne. Action Aid propose ainsi à ses internautes de créer leurs propres pages pour organiser la collecte de fonds localement. La Croix-Rouge, en France, parle de Web-bénévoles pour les internautes qui aident à faire connaître son action sur le Web. Ces sollicitations permettent aux donateurs de se sentir plus impliqués par l'organisation. Enfin, les organisations valorisent leurs plus généreux donateurs, par exemple en publiant leur nom dans leur rapport annuel ou, au RoyaumeUni, en inaugurant des plaques honorifiques dans des salles de musées. 


\section{Conclusion}

Cet article avait pour objectif de montrer les différences et les similitudes entre la France et le Royaume-Uni, mais aussi de tordre le cou à une idée reçue: le secteur associatif en France n'est pas moins développé quau Royaume-Uni (selon le montant global du secteur et le nombre d'organisations). Cependant, il est sans doute moins visible, notamment parce que les outils de collecte sont présents partout au Royaume-Uni: dans les grandes surfaces, au comptoir des magasins, au restaurant, dans la rue sous la forme de collecteurs, d'affichage, de magasins dédiés (les charity shops).

Si l'on ne retient que la collecte privée auprès des particuliers, les Britanniques sont plus nombreux à donner, pour un don moyen annuel très largement supérieur. Même en écartant les quelques donateurs très généreux, ce montant reste supérieur à la moyenne française.

Peut-être est-il parfois plus facile en Angleterre qu'en France d'ouvrir le dialogue pour collecter. Les Anglais donnent par exemple beaucoup en espèces lorsqu'ils sont sollicités dans la rue pour une cause. Ils ont une forte culture du don.

Avec la multiplication du nombre de structures et le développement d'organisations plus anciennes, la concurrence s'accroît et le marché de la générosité des particuliers ralentit au Royaume-Uni. Les charities doivent anticiper cette tendance. Les principaux enjeux deviennent alors l'innovation des outils de collecte et la diversification des publics visés: les grands donateurs, les entreprises avec le mécénat (lire en annexe). 


\section{Bibliographie}

Bruce I., 2005, Charity marketing, meeting need through customer focus, ICSA Guides Publishing.

Cerphi, 2005 et 2006, La générosité des Français, $10^{\mathrm{e}}$ et $11^{\mathrm{e}}$ éditions, www.cerphi.org. Cerphi, 2009, Le don d'argent des ménages aux associations et aux fondations.

Draperi J.-F., 2010, «Du marché public au public marché ", Recma, n 316.

Fondation de France, 2003, Regard international: la France du don comparée à ses proches voisins européens, étude, décembre.

Fondation de France (observatoire), 2006, Les générosités privées en France: tendances.

Fondation de France, 2009, Baromètre 2008 de la générosité en France.

Gallopel-Morvan K., Birambeau P., Larceneux F., Rieunier S., 2008, Marketing et communication des associations, Dunod.

Institut du CSA, 2007, Les Français et le financement des associations, novembre.

Institute for philanthropy, 2004, Who are the givers?

Juris Edition, 2010, Association et collectivités publiques, février.
Le Péchon N., Rieunier S., 2008, « Comment améliorer la collecte de fonds des Petits Frères des pauvres? Diagnostic, analyse des démarches des autres associations et recommandations ", mémoire du master en management des associations à l'IAE de Paris.

National Council for voluntary organisations (NCVO), 2007, The UK voluntary sector almanac, the state of the sector, 2007. NCVO-Charities Aid Foundation (CAF), 2005, Results of the 2004-05 survey of individual charitable giving in the UK.

Norton M., 2007, Need to know? Fundraising, Collins

Rieunier S., 2006, « Les 30-50 ans: une cible généreuse, mais critique vis-à-vis du mailing ", in La générosité des Français, Cerphi.

Sargeant A., 2005, Marketing management for nomprofit organizations, Oxford University Press.

Tchernonog V., 2007, « Les associations en France: poids, profils et évolutions ", colloque Addes. 


\section{Annexe Le mécénat d'entreprise}

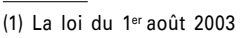
relative au mécénat offre aux entreprises une réduction d'impôt de $60 \%$ sur le montant de l'impôt des sociétés dans la limite de $0,5 \%$ du chiffre d'affaires.
Les entreprises britanniques, par le biais du mécénat et du parrainage, ont donné 1 milliard de livres en 2004-2005. S'il s'agit d'une somme importante, c'est peu au regard du total des revenus du secteur. Comme outre-Manche, le mécénat se développe en France, mais dans des proportions plus importantes, notamment grâce aux incitations fiscales proposées par la loi de $2003^{(1)}$, mais pas seulement, car $45 \%$ des entreprises mécènes n'en retirent pas d'avantages fiscaux. L'Admical indique sur son site (www.admical.org) que pour l'année 2008 les entreprises françaises se sont montrées très généreuses, avec près de 2,5 milliards d'euros, même si ce chiffre est à prendre avec précaution, car il a été estimé sur du déclaratif. Près de 30000 entreprises de plus de vingt salariés pratiqueraient le mécénat, qu’il soit financier, en nature (l'entreprise fournit des biens matériels à l'association, par exemple des espaces publicitaires gratuits, du matériel informatique...) ou de compétences (l'entreprise met son personnel à disposition pour mener à bien un projet ou une mission).

Cette " générosité " peut répondre à plusieurs motivations: améliorer l'image de l'entreprise en l'associant à un projet associatif, fidéliser les clients par le biais des événements glamour de l'organisation associative (Draperi, 2010), rencontrer de nouveaux contacts (d'éventuels clients...), associer le personnel en le mobilisant sur un projet (ce qui permet de rappeler les valeurs de l'entreprise) et bénéficier d'avantages fiscaux. Mais au-delà de ces aspects, l'association peut devenir un véritable partenaire professionnel pour l'entreprise. Les nouveaux fonds de dotation facilitent et formalisent ce type de partenariat, car ils ont la capacité d'associer plusieurs acteurs au moment de la création: associations, entreprises, particuliers, etc.

\section{Les fonds de dotation}

Créé par la loi de modernisation de l'économie du 4 août 2008 et complété par le décret du 11 février 2009, le fonds de dotation est une nouvelle personne morale sans but lucratif, calquée sur le modèle anglo-saxon des endowments funds. Il s'agit d'un organisme qui collecte des fonds privés (dons, donations, legs, mécénat...) afin de réaliser ou de soutenir financièrement des missions d'intérêt général. Il est présenté aujourd'hui comme un nouvel outil permettant de favoriser la collecte privée. Christine Lagarde, saluant la création des fonds de dotation, déclarait le 19 novembre 2008: "Dans le domaine des donations, il suffit d'ouvrir le cadenas réglementaire pour que l'argent coule enfin. Il était donc temps d'ouvrir ce dernier cadenas. " 
(2) D'après les informations disponibles sur le site du Journal officiel: www.journal-officiel. gouv.fr, fin mai 2010.
Fin mai 2010, près de trois cents fonds de dotation avaient été créés ${ }^{(2)}$. L'Ile-de-France est la région la plus dynamique, avec $50 \%$ des fonds de dotation créés; viennent ensuite Rhône-Alpes (8\%) et ProvenceAlpes-Côte d'Azur (6\%). Même si tous les secteurs d'activité sont concernés par cette vague de créations, le secteur culturel est le plus représenté, avec $18 \%$ des créations, puis viennent les actions caritatives (15\%), les interventions sociales (13\%), l'environnement, la préservation du patrimoine, l'aide à l'emploi, la santé, etc. Tous les acteurs possibles sont impliqués dans la création de fonds de dotation, mais il semble que les associations et les sociétés commerciales soient à l'origine du plus grand nombre de créations. Les motivations sont diverses et varient selon les acteurs: certaines associations souhaitent collecter des fonds sous la forme de donations et de legs, mais ne pourront jamais être reconnues d'utilité publique (par leur objet, leur nombre de membres, leur importance financière...), certains mécènes préfèrent créer un fonds de dotation plutôt qu'une fondation, car les fondateurs peuvent en garder le contrôle, etc.

Avec un fonds créé tous les cinq jours en France depuis plus d'un an, la dynamique des fonds de dotation est sans commune mesure avec celle des fondations. Il est encore un peu tôt pour savoir si les fonds de dotation vont permettre de développer fortement les financements privés, car la plupart des fonds créés jusqu'à présent n'ont qu'une faible dotation initiale. 